\title{
AG490 ameliorates early brain injury via inhibition of JAK2/STAT3-mediated regulation of HMGB1 in subarachnoid hemorrhage
}

\author{
JI-YANG AN ${ }^{1}$, HONG-GANG PANG ${ }^{2}$, TING-QIN HUANG ${ }^{2}$, JIN-NING SONG $^{2}$, \\ DAN-DONG $\mathrm{LI}^{2}$, YONG-LIN ZHAO ${ }^{2}$ and XU-DONG MA ${ }^{2}$ \\ ${ }^{1}$ Department of Neurosurgery, The First Affiliated Hospital of Zhengzhou University, Zhengzhou, Henan 450052; \\ ${ }^{2}$ Department of Neurosurgery, The First Affiliated Hospital of Xi'an Jiaotong University, Xi'an, Shaanxi 710061, P.R. China
}

Received October 25, 2015; Accepted February 27, 2017

DOI: $10.3892 /$ etm.2017.5539

\begin{abstract}
High mobility group box 1 (HMGB1) is a classic damage-associated molecular pattern that has an important role in the pathological inflammatory response. In vitro studies have demonstrated that the Janus kinase 2 (JAK2)/signal transducer and activator of transcription 3 (STAT3) signaling pathway is involved in the regulation of HMGB1 expression, mediating the inflammatory response. Therefore, the purpose of the present study was to evaluate JAK2/STAT3 pathway involvement in the subarachnoid hemorrhage (SAH)-dependent regulation of HMGB1, using an in vivo rat model. A SAH model was established by endovascular perforation. Western blotting, immunohistochemistry and immunofluorescence were used to analyze HMGB1 expression after SAH. In addition, the effects of AG490 after SAH on JAK2/STAT3 phosphorylation, HMGB1 expression and brain damage were evaluated. The results of the present study demonstrated that JAK2/STAT3 was significantly phosphorylated $(\mathrm{P}<0.05)$ and the total HMGB1 protein level was significantly increased $(\mathrm{P}<0.05)$ after SAH. In addition, the cytosolic HMGB1 level after SAH demonstrated an initial increase followed by a decrease to the control level, while the nuclear HMGB1 level after SAH demonstrated the opposite trend, with an initial decrease and subsequent increase. AG490 administration after SAH significantly inhibited JAK2/STAT3 phosphorylation $(\mathrm{P}<0.05)$, suppressed the expression and translocation of HMGB1, reduced cortical apoptosis, brain edema and neurological deficits. These results demonstrated the involvement of the JAK2/STAT3 pathway in HMGB1 regulation after SAH.
\end{abstract}

Correspondence to: Dr Ji-Yang An, Department of Neurosurgery, The First Affiliated Hospital of Zhengzhou University, 1 Jianshe Road, Zhengzhou, Henan 450052, P.R. China

E-mail: anjiyang@163.com

Key words: subarachnoid hemorrhage, Janus kinase 2/signal transducer and activator of transcription 3 signaling pathway, high mobility group box 1, AG490, neuronal apoptosis

\section{Introduction}

Subarachnoid hemorrhage ( $\mathrm{SAH})$ is a devastating disease associated with neurological deficits and mortality. After decades of intense research focused on the pathological events that occur after SAH, vasospasm is considered the predominant cause of neuronal death and subsequent neurological dysfunction after SAH (1). However, prevention of cerebral vasospasm has a limited effect on improving the outcome for patients after SAH (2). Thus, other potential mechanisms, which may have crucial roles in pathological processes after $\mathrm{SAH}$, should be considered. Inflammation due to debris and metabolites from necrotic or activated cells is particularly prominent after $\mathrm{SAH}$, and it may be involved in early brain damage after $\mathrm{SAH}(3,4)$.

High mobility group box 1 (HMGB1) is a classic damage-associated molecular pattern that is usually located in the nucleus as a DNA-binding protein and is involved in the stabilization of nucleosome formation and gene transcription (5). In the central nervous system, HMGB1 is widely expressed in neurons and glia, and may be released by necrotic neurons and activated microglia or astrocytes in rats following cerebral ischemia or traumatic brain injuries $(6,7)$. When released into the extracellular space, HMGB1 may promote the expression of multiple cytokines, including tumor necrosis factor (TNF)- $\alpha$, interleukin (IL)-1 $\beta$ and IL-6 $(8,9)$. Previous studies have demonstrated significantly elevated HMGB1 levels in the cerebral spinal fluid of patients who have experienced $\mathrm{SAH}$, and it has been associated with the disease outcome (10). The mechanisms regulating the expression of HMGB1 after SAH have not been fully elucidated. An in vitro study demonstrated that the Janus kinase (JAK)/signal transducer and activator of transcription (STAT) pathway may mediate HMGB1 expression and inflammatory effects (11). The JAK/STAT cascade is an important inflammatory signaling pathway in mediating immune responses, it is widely expressed in the brain and has important roles in maintaining the balance between pro-inflammation and anti-inflammation (12-14). Therefore, JAK2/STAT3 may be involved in the regulation of HMGB1 expression after SAH. To the best of our knowledge, the role of JAK2/STAT3 in SAH-induced HMGB1 translocation has not been clarified. Therefore, the aim of the present study was 
to evaluate whether the JAK2/STAT3 pathway is involved in the SAH-dependent regulation of HMGB1, using an in vivo rat model.

\section{Materials and methods}

Animals and experimental groups. A total of 105 male Sprague-Dawley rats weighing 320 \pm 26 g (2.0-2.5 months old) were purchased from the Laboratory Animal Center of Xi'an Jiaotong University (Xi'an, China). Animals were housed at $25^{\circ} \mathrm{C}$ and $50 \%$ humidity with a $12 \mathrm{~h}$ light/dark cycle and free access to water and food. The animals were randomly divided into four groups: Sham $(n=18)$, SAH $(n=45), S A H+$ vehicle (dimethyl sulfoxide; DMSO; $n=21)$ and SAH + AG490 (n=21) groups. The experimental procedures were approved by the Biomedical Ethics Committee of the Medical School of Xi'an Jiaotong University.

Experimental model of SAH and drug administration. Rats were anesthetized with an intraperitoneal injection of $10 \%$ chloral hydrate $(0.35 \mathrm{ml} / \mathrm{kg})$ and placed in a prone position. A rat model of SAH was induced by endovascular perforation, according to a study by Bederson et al (15). Rats in the $\mathrm{SAH}+\mathrm{AG} 490$ and SAH + vehicle groups were treated with 2 ml AG490 (5 mM; Sigma-Aldrich; Merck KGaA, Darmstadt, Germany) or DMSO, respectively, via intraventricular injection 30 min prior to SAH (16). The injection site was $1.0 \mathrm{~mm}$ caudal to bregma, $1.5 \mathrm{~mm}$ lateral to midline and $4.0 \mathrm{~mm}$ deep from the dura. A rectal probe monitored the body temperature and a heating lamp maintained normothermia during the entire procedure.

Neurological severity scores (NSS). Neurobehavioral functions were evaluated via NSS tests in the four groups using the modified Garcia test (17) at 1 day post-SAH induction by two researchers blinded to the groupings.

Brain water content $(B W C)$ measurement. On day 1 following $\mathrm{SAH}$ induction, 5 rats from each group were sacrificed to evaluate BWC using the standard wet-dry method. Following sacrifice, brains were quickly removed and weighed for wet weight determination. Subsequently, brains were dried in an oven at $105^{\circ} \mathrm{C}$ for $72 \mathrm{~h}$ and the dry weight was evaluated. The following formula was used to calculate the brain water content: (wet weight-dry weight)/wet weight x $100 \%$.

Immunohistochemistry (IHC). Euthanasia was conducted at $6 \mathrm{~h}, 1 \mathrm{~d}$ and 3 days post-SAH. Rats in the Sham group were euthanized at 1 day after operation. A total of 5 rats in each group were sacrificed by decapitation under anesthesia and perfused with $250 \mathrm{ml}$ of normal saline followed by $400 \mathrm{ml}$ of $4 \%$ paraformaldehyde in $0.01 \mathrm{M}$ PBS. The whole brains were removed and fixed in $4 \%$ paraformaldehyde solution at $4^{\circ} \mathrm{C}$ for $24 \mathrm{~h}$, embedded in paraffin and cut into $15-\mu \mathrm{m}$ serial sections. Routine hematoxylin and eosin staining was performed (hematoxylin staining for $5 \mathrm{~min}$ and eosin staining for $2 \mathrm{~min}$, both at room temperature), and IHC staining was performed to detect HMGB1 expression after SAH. Sections were deparaffinized with xylene and rehydrated by immersion in a graded series of ethanol. Endogenous peroxidase activity was blocked using $3 \% \mathrm{H}_{2} \mathrm{O}_{2}$ for 5 min, followed by citric acid buffer ( $\mathrm{pH}$ 6.0) microwave antigen retrieval. Nonspecific protein binding was blocked by $2 \mathrm{~h}$ incubation in $5 \%$ bovine serum at room temperature. Sections were incubated with rabbit HMGB1 monoclonal antibody (1:200; ab190377; Abcam, Cambridge, MA, USA) as the primary antibody for $1 \mathrm{~h}$ at room temperature and washed with PBS 3 times for $5 \mathrm{~min}$. Subsequently, the sections were incubated with horseradish peroxidase (HRP)-conjugated immunoglobulin (1:500; cat. no. sc-2357; Santa Cruz Biotechnology, Inc., Dallas, TX, USA) as the secondary antibody for $60 \mathrm{~min}$ at room temperature. 3,3'-diaminobenzidine was used as a chromogen and the sections were counterstained with hematoxylin for $5 \mathrm{~min}$ at room temperature. Microscopic observation was performed by an experienced pathologist blinded to the experimental conditions using the Leica-Q550CW image acquisition and analysis system (Leica Microsystems GmbH, Wetzlar, Germany). The immunoreactivity of all of the molecular markers was analyzed using Image-Pro Plus 6.0 software (Media Cybernetics, Inc., Rockville, MD, USA) in five microscopic fields (magnification, x200).

Confocal microscopy. Confocal microscopy was used to observe HMGB1 translocation after SAH. Brain sections were prepared as described in the immunohistochemistry. Following rehydration, sections were incubated with $0.2 \%$ Triton X-100 in PBS for $5 \mathrm{~min}$ at room temperature and washed with PBS 3 times for $5 \mathrm{~min}$. Sections were placed in $10 \mathrm{mmol} / 1$ citrate buffer ( $\mathrm{pH}$ 6.0) and heated in the microwave oven at $95^{\circ} \mathrm{C}$ for $30 \mathrm{~min}$. Sections were cooled at room temperature for $20 \mathrm{~min}$ and rinsed in PBS. Nonspecific protein binding was blocked by incubation in $5 \%$ bovine serum for $40 \mathrm{~min}$. Sections were incubated with primary rabbit anti-HMGB1 (1:200; ab190377; Abcam) overnight at $4^{\circ} \mathrm{C}$, followed by washing with PBS 3 times for $5 \mathrm{~min}$. Sections were incubated with goat anti-rabbit secondary antibody (1:100) conjugated with fluorescein isothiocyanate and tetramethylrhodamine (cat. no.ZF-0311; Beijing Zhongshan Golden Bridge Biotechnology, Beijing, China) at $37^{\circ} \mathrm{C}$ for $1 \mathrm{~h}$. The cell nucleus was stained with 4,6'-diamidino-2-phenylindole, dihydrochloride (1:1,000; Sigma-Aldrich; Merck KGaA). Immunofluorescence was detected using a laser scanning confocal microscope (Olympus FV10-ASW; Olympus Corp., Tokyo, Japan).

Western blot analysis. Phosphorylation of JAK2/STAT3 and HMGB1expression after SAH were evaluated in the rats of each group. Brain tissues were dissolved in lysis buffer $(50 \mathrm{mM}$ Tris-HCl, 2 mM EDTA and 1\% Nonidet). Nucleoprotein and cytoplasmic protein were extracted using a nuclear and cytoplasmic protein extraction kit (Beyotime Institute of Biotechnology, Haimen, China), and protein concentrations were determined using Bradford reagent (Beyotime Institute of Biotechnology), according to the manufacturers' instructions. Protein samples $(50 \mu \mathrm{g})$ were separated by $10 \%$ SDS-PAGE and transferred to a polyvinylidene difluoride membrane. Following incubation for $2 \mathrm{~h}$ in a blocking solution [5\% non-fat milk in $20 \mathrm{mM}$ Tris- $\mathrm{HCl}, 150 \mathrm{mM} \mathrm{NaCl}, 0.1 \%$ Tween-20 (TBST), at room temperature], the membrane was incubated with the following antibodies: Rabbit anti-HMGB1 monoclonal antibody (1:2,000; ab190377; Abcam), rabbit anti-Lamin 

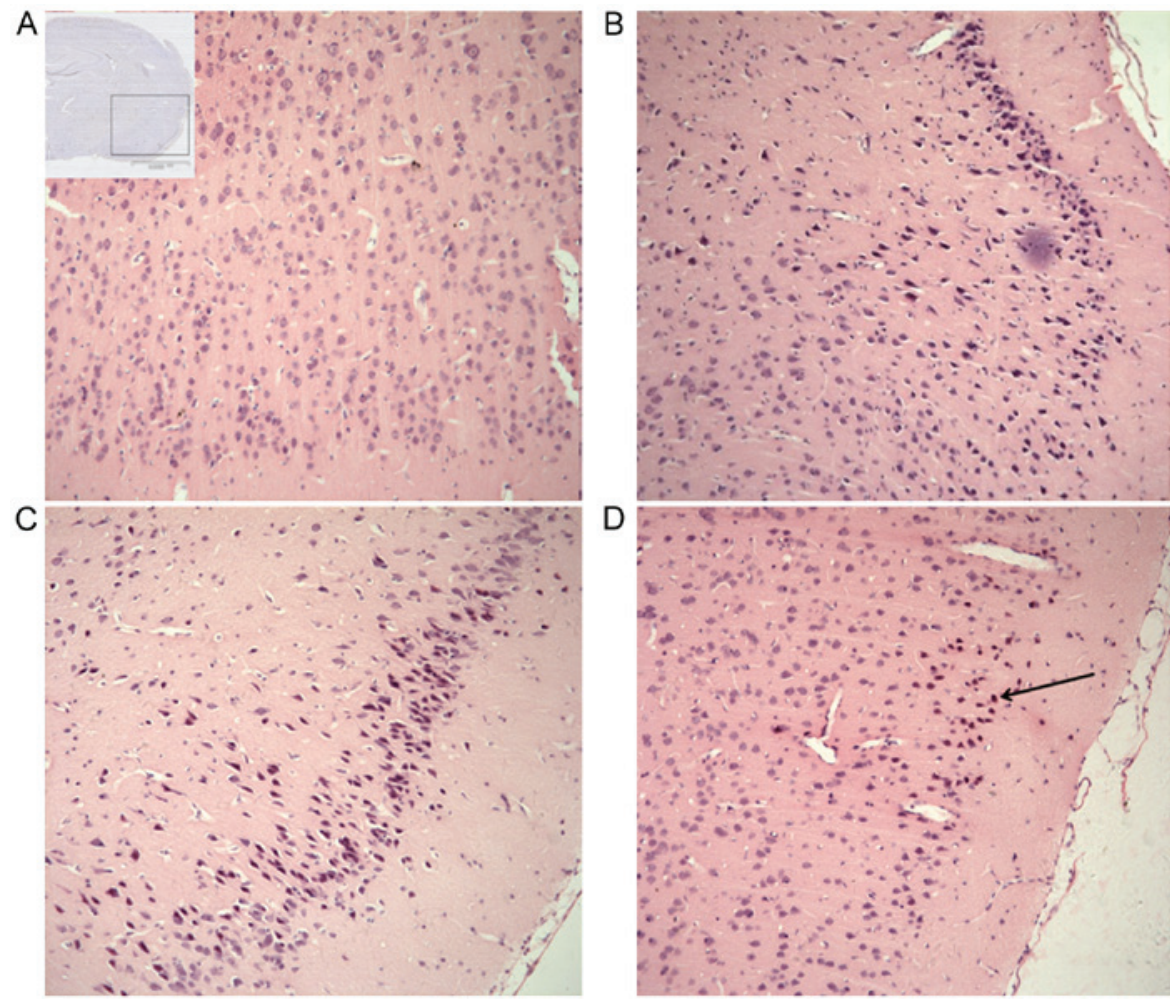

Figure 1. Histopathological changes following SAH. (A) Inset: The site of observation, in which the presence of blood is clear. (A) Normal rat brain samples from the sham group demonstrated normal morphology and clear neuron and glial cell structure. Samples harvested (B) 6 h, (C) 1 day and (D) 3 days after SAH induction. Injured cells were observed, characterized by cell shrinkage, pyknosis and chromatin fragmentation (arrow), which were abundantly present after SAH. Scale bar, $200 \mu \mathrm{m}$; hematoxylin-eosin staining. Magnification, x100.

B1 monoclonal antibody (1:2,000; ab16048; Abcam), mouse anti- $\beta$-actin polyclonal antibody $(1: 5,000$; SC-47778; Santa Cruz Biotechnology, Inc.), rabbit anti-p-JAK2 monoclonal antibody (1:2,000; cat. no. 3776; Cell Signaling Technology, Danvers, MA,USA), rabbit anti-p-STAT3 monoclonal antibody (1:2,000; cat. no. 9134; Cell Signaling Technology) overnight at $4^{\circ} \mathrm{C}$. Following extensive washing with TBST, membranes were incubated with HRP-conjugated anti-rabbit/mouse secondary antibodies (1:5,000; cat. no. 7074/7076; Cell Signaling Technology) for $1 \mathrm{~h}$ at room temperature. Specific bands were detected using a ECL Western Blotting Detection System (cat. no. WBKLS0100; EMD Millipore, Billerica, MA, USA). Quantitative analysis was performed using Gel-Pro Analyzer v.3.1 software (Media Cybernetics, Inc., Rockville, MD, USA).

Terminal deoxynucleotidyl transferase dUTP nick-end labeling (TUNEL) staining. DNA fragmentation in the cell nuclei of brain tissue sections was determined by TUNEL staining using the DeadEnd colorimetric TUNEL system detection kit (cat. no. G7130; Promega Corporation, Madison, WI, USA), according to the manufacturer's protocol. Brain sections were prepared as described in the immunohistochemistry. The sections were initially deparaffinized with xylene, rehydrated using a graded series of ethanol, rinsed for $15 \mathrm{~min}$ in $0.1 \mathrm{M}$ PBS and then treated with $20 \mu \mathrm{g} / \mathrm{ml}$ of Proteinase $\mathrm{K}$ for $20 \mathrm{~min}$ at room temperature. Samples were treated with $3 \% \mathrm{H}_{2} \mathrm{O}_{2}$ in methanol for $20 \mathrm{~min}$ to inactivate endogenous peroxidase. Following washing with PBS, specimens were incubated in rTdT reaction mixture (100 $\mu \mathrm{l}$; combining
$98 \mu 1$ of Equilibration Buffer, $1 \mu 1$ of Biotinylated Nucleotide Mix and $1 \mu \mathrm{l}$ of rTdT Enzyme) at $4^{\circ} \mathrm{C}$ overnight. Following incubation, sections were rinsed in PBS and incubated with horseradish peroxidase (dilution, 1:500) for $30 \mathrm{~min}$ at room temperature. Sections were washed extensively with PBS for $5 \mathrm{~min}$ and treated with DAB solution (30 mg DAB and $200 \mu \mathrm{l}$ $\mathrm{H}_{2} \mathrm{O}_{2} / 100 \mathrm{ml}$ PBS) for $10 \mathrm{~min}$ at room temperature in the dark. Following washing under running water, all the sections were counterstained with hematoxylin for $30 \mathrm{sec}$. Finally, the sections were dehydrated in graded ethanol, cleared with xylene and mounted with a cover slip. Using this method, apoptotic nuclei were identified by the presence dark brown staining.

Statistical analysis. Data were presented as the mean \pm standard deviation. SPSS v.13.0 (SPSS, Inc., Chicago, IL, USA) was used for statistical analyses. Comparison between different groups was made by one-way analysis of variance (ANOVA), followed by Tukey's multiple comparisons test if a significant difference was demonstrated by ANOVA. $\mathrm{P}<0.05$ was considered to indicate a statistically significant difference.

\section{Results}

Histopathological changes after SAH. Hematoxylin-eosin staining was used to observe the histopathological changes after SAH. The cortex tissue from the sham group demonstrated a normal morphology and clear neuron and glial cell structure (Fig. 1A). Injured neuronal cells appeared at $6 \mathrm{~h}$ after 

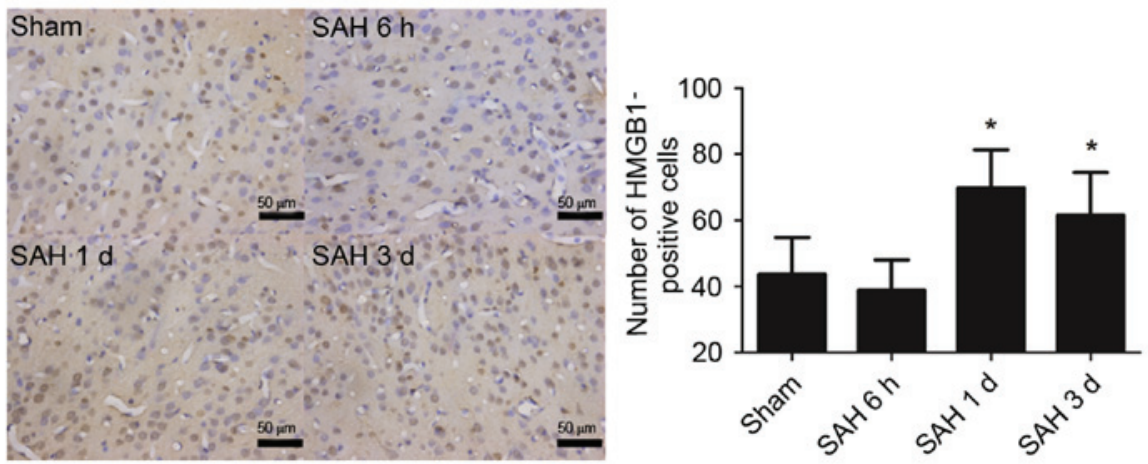

Figure 2. HMGB1 expression following SAH. HMGB1 was widely expressed by cells in the normal (Sham) rat brain and predominantly stored in the nucleus. The number of HMGB1-expressing cells decreased at $6 \mathrm{~h}$ after SAH compared with the Sham group. At days 1 and 3 after SAH, the number of HMGB1 positive immuno-stained cells significantly increased compared with the Sham group. Data are presented as the mean \pm standard deviation. ${ }^{*} \mathrm{P}<0.05 \mathrm{vs}$. the Sham. Scale bar, $50 \mu \mathrm{m}$. Magnification, x200. SAH, subarachnoid hemorrhage; HMGB1, high mobility group box 1; SAH $6 \mathrm{~h}, 6 \mathrm{~h}$ after SAH induction; SAH $1 \mathrm{~d}, 1$ day after SAH induction; SAH 3 d, 3 days after SAH induction.

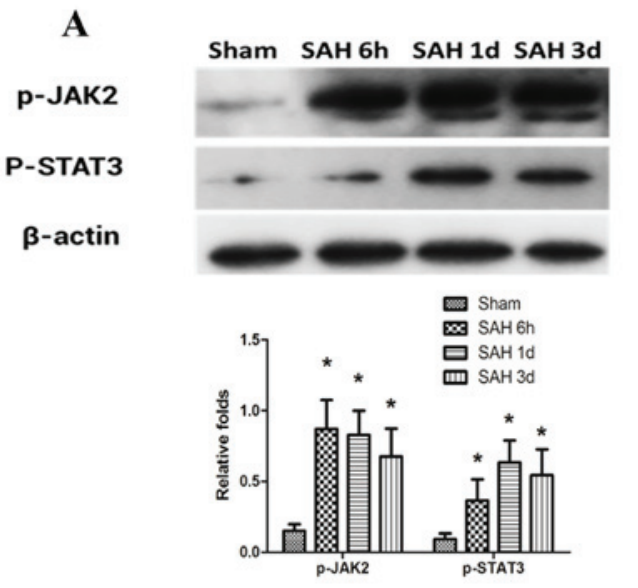

C

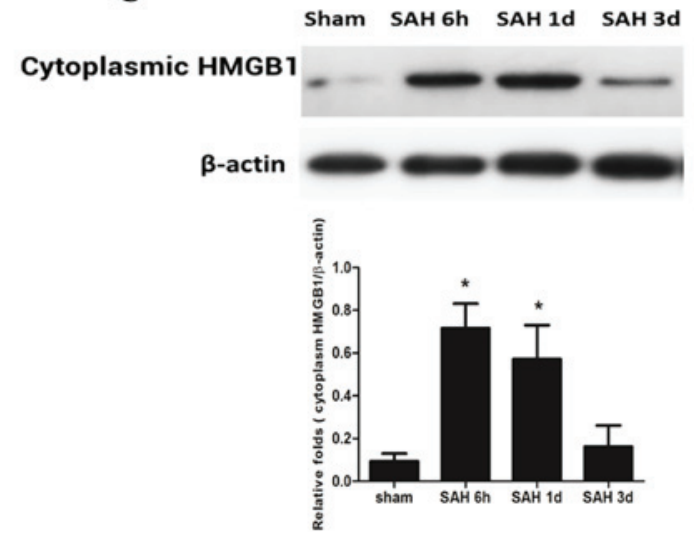

B

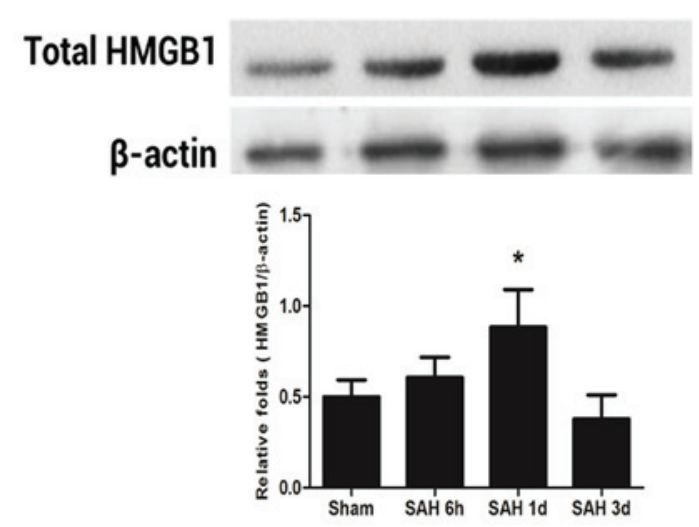

D
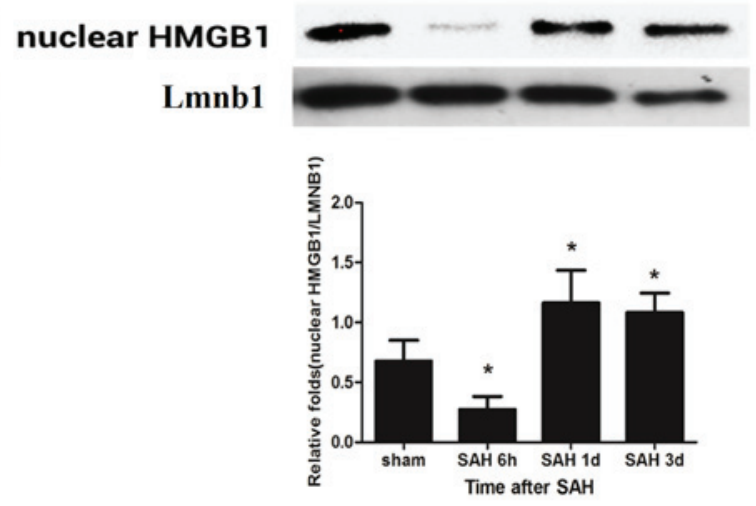

Figure 3. HMGB1 dynamic change and JAK2/STAT3 phosphorylation after SAH. (A) The levels of phosphorylated JAK2 and STAT3 relative to $\beta$-actin were significantly increased at $6 \mathrm{~h}, 1$ day and 3 days after SAH compared with the Sham group. (B) No significant increase of total HMGB1 expression was observed at $6 \mathrm{~h}$ after SAH; however, a significant increase was demonstrated at day 1 compared with the Sham group. The level then decreased to the baseline level at day 3. (C) Cytoplasmic HMGB1 level significantly increased at $6 \mathrm{~h}$ and 1 day after SAH compared with the Sham group; however, the level decreased at day 3. (D) Nuclear HMGB1 significantly decreased at $6 \mathrm{~h}$ after SAH compared with the Sham group; however, this level increased at day 1 and day 3 compared with the Sham group. $\beta$-actin and LMNB1 were used as internal references. Data are presented as the mean \pm standard deviation. " $\mathrm{P}<0.05$ vs. the Sham group. HMGB1, high mobility group box 1; JAK2/STAT3, Janus kinase 2/signal transducer and activator of transcription 3; SAH, subarachnoid hemorrhage; SAH $6 \mathrm{~h}$, $6 \mathrm{~h}$ after SAH induction; SAH 1 d, 1 day after SAH induction; SAH 3 d, 3 days after SAH induction; p, phosphorylated; LMNB1, Lamin B1.

SAH, characterized by cell shrinkage, pyknosis and chromatin fragmentation, all effects gradually increased with increasing time after SAH (Fig. 1B-D).
Cortical HMGB1 expression after SAH. IHC staining demonstrated HMGB1 was widely expressed by cells in the cortex of the sham group. This expression decreased slightly at $6 \mathrm{~h}$ after 
A
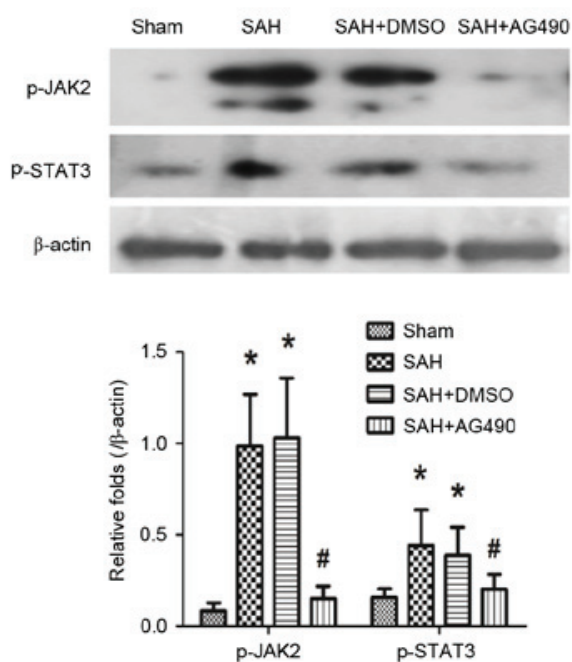

C

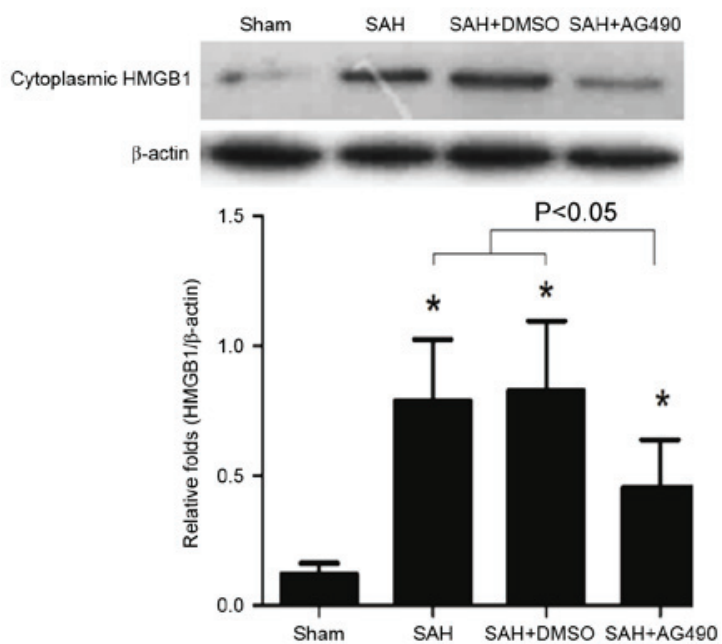

B
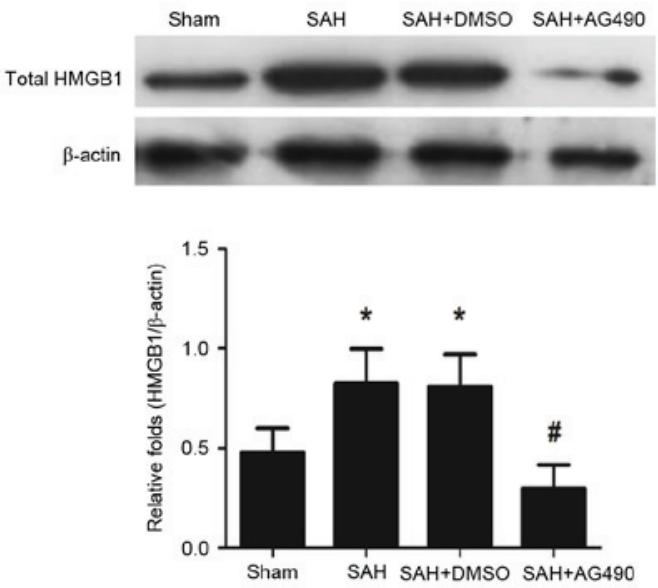

D
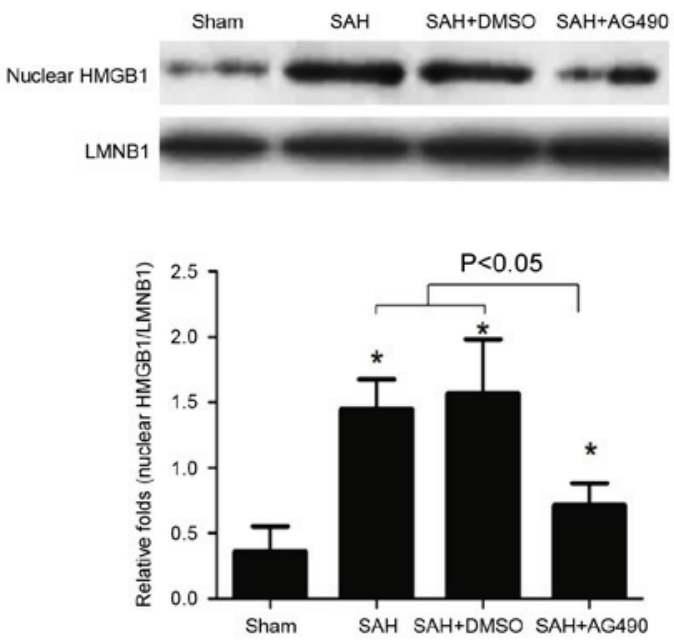

Figure 4. Regulatory effects of AG490. (A) AG490 significantly inhibited JAK2/STAT3 phosphorylation after SAH compared with the SAH and SAH + DMSO groups. (B) Total, (C) cytoplasmic and (D) nuclear HMGB1 expression was inhibited by AG490 administration following SAH compared with the SAH and $\mathrm{SAH}+\mathrm{DMSO}$ groups. $\beta$-actin and LMNB1 were used as internal references. Data are presented as the mean \pm standard deviation. ${ }^{*} \mathrm{P}<0.05$ vs. the Sham group; " $\mathrm{P}<0.05$ vs. the $\mathrm{SAH}$ and $\mathrm{SAH}+\mathrm{DMSO}$ groups. JAK2/STAT3, Janus kinase 2/signal transducer and activator of transcription 3; SAH, subarachnoid hemorrhage; DMSO, dimethyl sulfoxide; LMNB1, Lamin B1.

SAH; however, this difference was not statistically significant. At days 1 and 3 after SAH, the number of HMGB1-positive cells significantly increased compared with the sham group (P<0.05; Fig. 2).

JAK2/STAT3 phosphorylation and HMGB1 expression after $S A H$. The phosphorylation of JAK2/STAT3 was low in the sham group. The level of JAK2/STAT3 phosphorylation began to increase at $6 \mathrm{~h}$ after $\mathrm{SAH}$, and at days 1 and 3 this level remained high. The level of JAK2/STAT3 phosphorylation was significantly higher at $6 \mathrm{~h}$, and days 1 and 3 after SAH induction when compared with the sham group $(\mathrm{P}<0.05$; Fig. 3A). Western blotting was used to detect HMGB1 protein level in the cortex after SAH. Firstly, total HMGB1 protein expression level at $6 \mathrm{~h}, 1$ and 3 days after SAH was detected, followed by the detection of the cytoplasmic and nuclear HMGB1 levels. The total HMGB1 protein expression level was significantly increased at day 1 after SAH compared with the sham group $(\mathrm{P}<0.05)$; however, at $6 \mathrm{~h}$ and day 3 , this difference was not significant (Fig. 3B). The cytoplasmic HMGB1 level significantly increased at $6 \mathrm{~h}$ and day 1 after SAH when compared with the sham group $(\mathrm{P}<0.05)$; however, it decreased to a level similar to the sham group at day 3 after SAH (Fig. 3C). The nuclear HMGB1 level significantly decreased at $6 \mathrm{~h}$ after SAH $(\mathrm{P}<0.05)$, and significantly increased at days 1 and 3 after $\mathrm{SAH}$, as compared with the sham group $(\mathrm{P}<0.05$; Fig. 3D).

Effect of AG490 on JAK2/STAT3 phosphorylation and $H M G B 1$ expression. According to western blot analysis, HMGB1 protein expression peaked at day 1 after SAH. Thus, the effects of AG490 were analyzed at day 1 after SAH. Results demonstrated that JAK2/STAT3 phosphorylation was significantly inhibited by AG490 administration compared with the SAH and SAH + DMSO groups ( $\mathrm{P}<0.05$; Fig. 4A). The total HMGB1 level was also significantly attenuated in the $\mathrm{SAH}+\mathrm{AG} 490$ group compared with the SAH and $\mathrm{SAH}+\mathrm{DMSO}$ groups $(\mathrm{P}<0.05$; Fig. 4B). Cytoplasmic and 


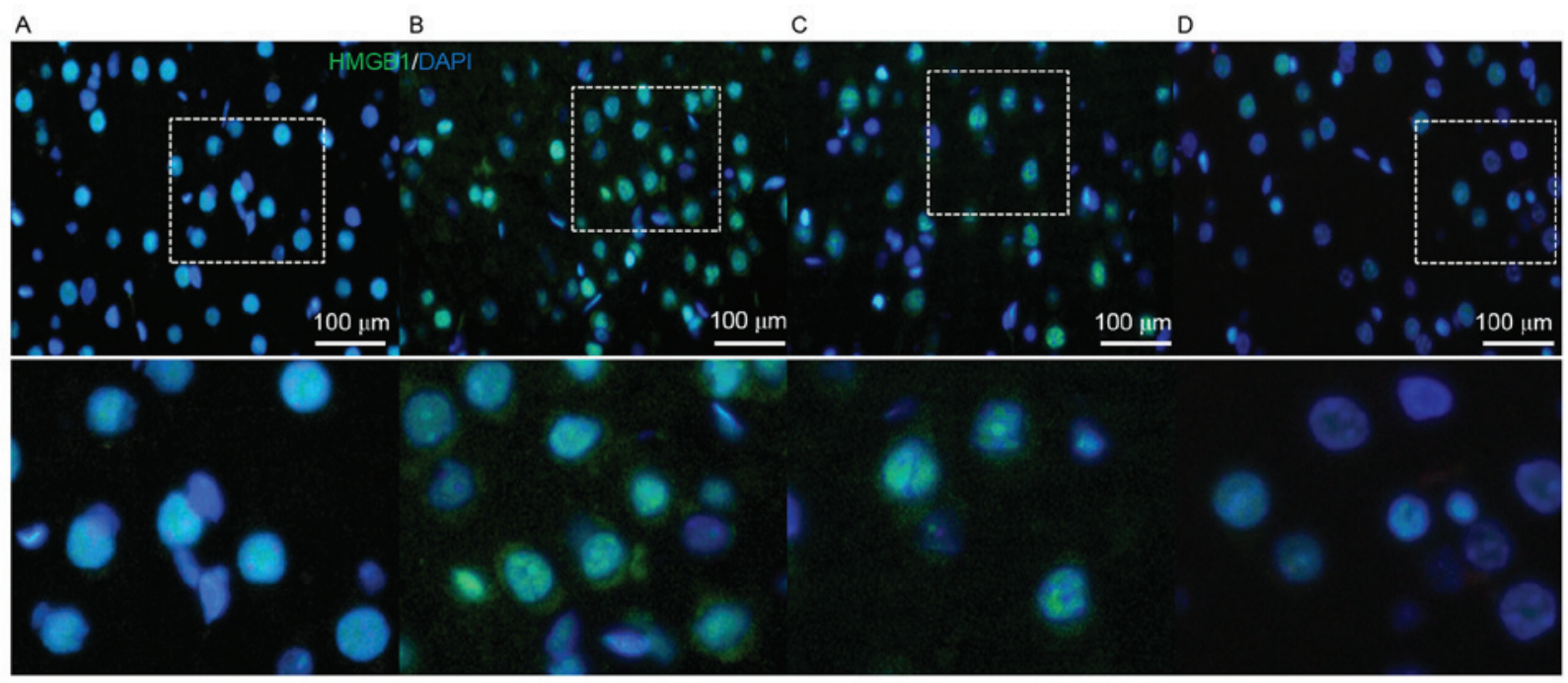

Figure 5. Immunofluorescent image of HMGB1 translocation (green) after the administration of AG490 on day 1 after SAH. (A) In the Sham group, HMGB1 was predominantly stored in the nucleus and no HMBG1 expression was demonstrated in the cytosol. The (B) SAH and (C) SAH + DMSO groups demonstrated positive HMGB1 immunofluorescence in the periphery of the nucleus without or with DMSO administration, respectively, which indicated that HMGB1 translocated into the cytosol after SAH. (D) The SAH + AG490 group demonstrated that AG490 administration inhibited HMGB1 nuclear expression, as well as its translocation into the cytosol. Scale bar, $100 \mu \mathrm{m}$. Magnification, x400. HMGB1, high mobility group box 1; SAH, subarachnoid hemorrhage; DMSO, dimethyl sulfoxide; DAPI, 4,6'-diamidino-2-phenylindole, dihydrochloride.
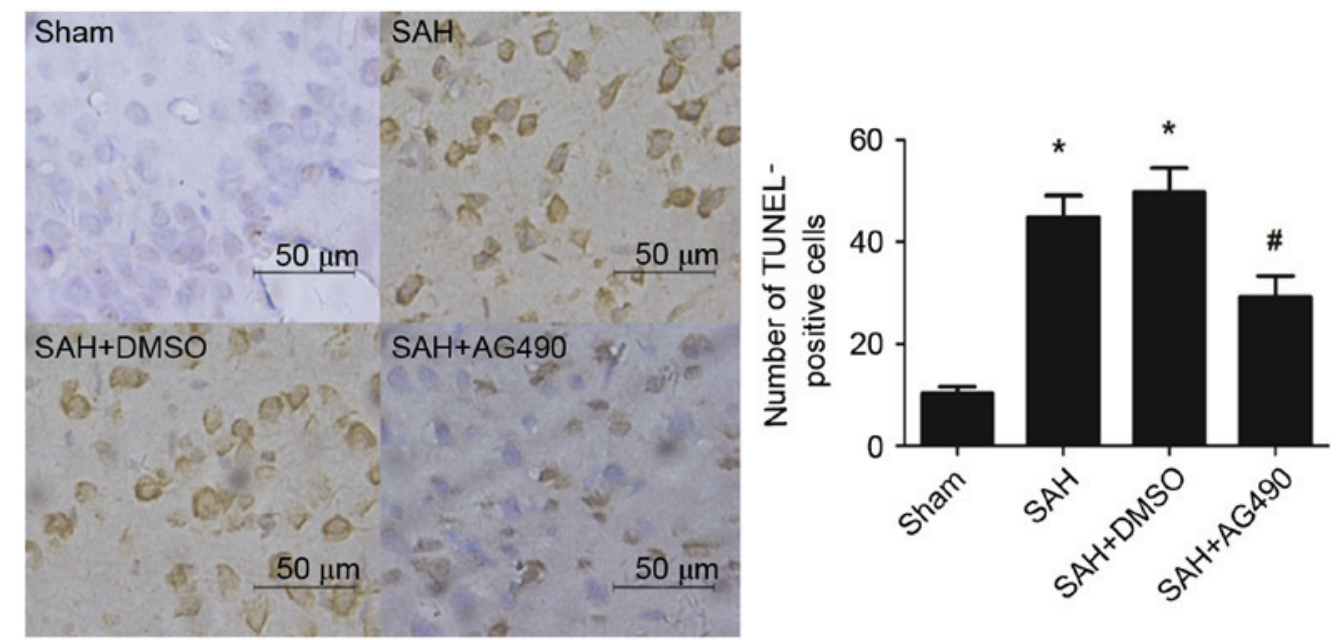

Figure 6. Effects of AG490 on neuronal apoptosis after SAH detected by TUNEL staining. Apoptotic cells are stained brown. (A) No apoptosis was observed in the cortex of rat brains from the Sham group. The (B) SAH and (C) SAH + DMSO groups demonstrated a significant increase in the number of apoptotic cells after SAH without or with DMSO administration, respectively, compared with the Sham group. (D) AG490 administration inhibited neuronal apoptosis after SAH compared with the SAH + DMSO group. Data are presented as the mean \pm standard deviation. ${ }^{*} \mathrm{P}<0.05$ vs. the Sham group; ${ }^{*} \mathrm{P}<0.05$ vs. the $\mathrm{SAH}$ and SAH + DMSO groups. Scale bar, $50 \mu \mathrm{m}$. Magnification, x400. SAH, subarachnoid hemorrhage; TUNEL, terminal deoxynucleotidyl transferase dUTP nick-end labeling; DMSO, dimethyl sulfoxide.

nuclear HMGB1 protein levels were also analyzed after AG490 administration following $\mathrm{SAH}$, and the results demonstrated that AG490 significantly inhibited both the cytoplasmic and nuclear HMGB1 expression compared with the SAH and $\mathrm{SAH}+\mathrm{DMSO}$ groups $(\mathrm{P}<0.05$; Fig. $4 \mathrm{C}$ and $\mathrm{D})$. These results suggested that JAK2/STAT3 may be involved in the regulation of HMGB1 expression after SAH.

AG490 effect on HMGB1 translocation after SAH. HMGB1 is able to translocate from the nucleus to the cytosol (11). AG490 may inhibit the expression of HMGB1 after SAH; however, its effects on the translocation of HMGB1 require clarification.
Thus, HMGB1 distribution was examined by immunofluorescence using a confocal microscope. The results demonstrated marked HMGB1 translocation from the nucleus to the cytosol in the SAH and SAH + DMSO groups. However, no obvious translocation was observed in the SAH + AG490 group, and nuclear HMGB1 also decreased (Fig. 5). These results suggested that AG490 may inhibit HMGB1 expression and suppress its cytosolic translocation.

Effect of AG490 on cortical apoptosis after SAH. Few TUNEL-positive apoptotic cells were observed in the sham group. After SAH, the number of apoptotic cells in the cortex 
A

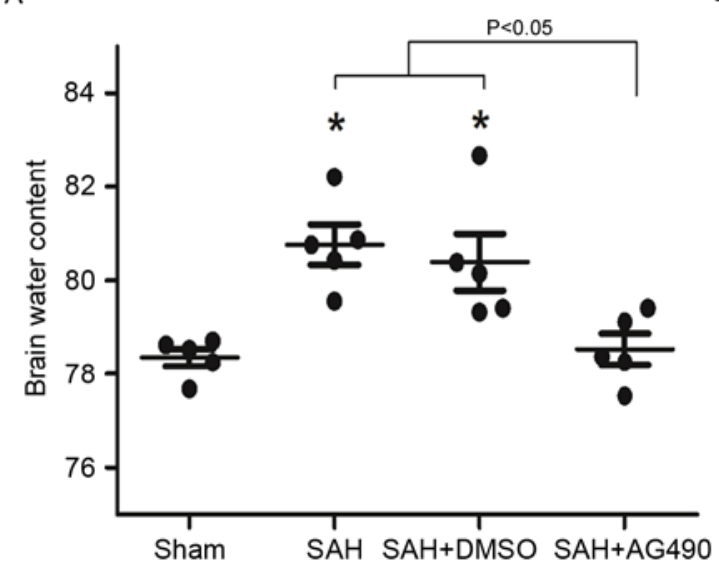

B

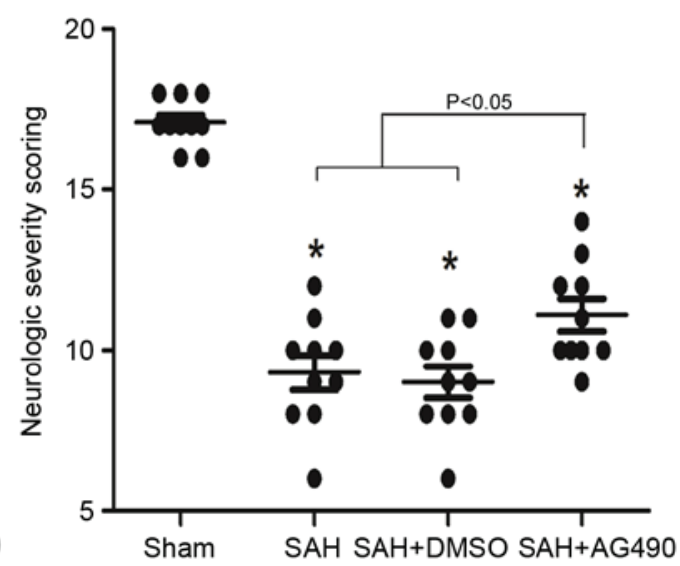

Figure 7. Brain edema and neurological deficit evaluation following administration of AG490 at day 1 after SAH. (A) Brain water content was significantly higher in the SAH and SAH + DMSO groups compared with the Sham group at day 1 after SAH. AG490 administration significantly reduced brain edema after SAH compared with the SAH and SAH + DMSO groups; (B) Neurological scoring was significantly lower in the SAH and SAH + DMSO groups compared with the Sham group at days 1, 3 and 7 after SAH. However, neurological deficits significantly improved in the SAH + AG490 group compared with the SAH and SAH + DMSO groups at days 1, 3 and 7 after SAH. Data are presented as the mean \pm standard deviation. ${ }^{*} \mathrm{P}<0.05$ vs. the SAH and SAH + DMSO groups. SAH, subarachnoid hemorrhage; DMSO, dimethyl sulfoxide.

significantly increased compared with that in sham group $(\mathrm{P}<0.05$; Fig. 6). No statistically significant difference was found between the SAH and SAH + DMSO groups. However, the number of apoptotic cells significantly decreased in the $\mathrm{SAH}+\mathrm{AG} 490$ group compared with the SAH + DMSO group $(\mathrm{P}<0.05$; Fig. 6). These results suggested that AG490 administration may reduce neuronal cells apoptosis in the brain as a result of SAH.

Effect of AG490 on brain edema and neurological deficits after $S A H$. BWC was significantly higher at day 1 in the SAH and $\mathrm{SAH}+\mathrm{DMSO}$ groups compared with sham group $(\mathrm{P}<0.05)$, while no significant difference was demonstrated between the SAH and SAH + DMSO groups at each time point. AG490 administration significantly reduced BWC compared with the $\mathrm{SAH}$ and $\mathrm{SAH}+\mathrm{DMSO}$ groups $(\mathrm{P}<0.05$; Fig. $7 \mathrm{~A})$. In the $\mathrm{SAH}$ and SAH + DMSO groups, NSS was significantly reduced at day 1 after SAH compared with the sham group $(\mathrm{P}<0.05)$. However, AG490 administration significantly improved the neurological function in the SAH + AG490 group compared with the SAH and SAH + DMSO groups $(\mathrm{P}<0.05$; Fig. 7B).

\section{Discussion}

Delayed vasospasm, which may result in microcirculatory dysfunction and cerebral ischemia following $\mathrm{SAH}$, is considered the most common risk complication of SAH (18). However, prevention of vasospasm with nimodipine has demonstrated limited effects on the improvement of patients who have experienced SAH (19).

Therefore, the present study focused on the inflammatory response associated with HMGB1 in early brain injury occurring in the first 3 days after $\mathrm{SAH}$, which has been demonstrated to have an important role in brain damage after SAH (20).

Previous studies have demonstrated that HMGB1 levels were significantly increased after SAH induced by cisterna magna injection $(21,22)$. In the present study, it was demonstrated that total HMGB1 expression initially increased at day 1 after $\mathrm{SAH}$, and subsequently decreased to the basic level at day 3. The cytosolic HMGB1 level significantly increased after SAH, and nuclear HMGB1 decreased at $6 \mathrm{~h}$, followed by an increase at days 1 and 3 after SAH. These results suggested that transcriptional expression of HMGB1 was activated at $6 \mathrm{~h}$ after SAH, and the early cytosolic HMGB1 increase was predominantly due to the translocation of nuclear HMGB1. Previous research has indicated that HMGB1 may be secreted from the nucleus to the cytosol by monocytes via a non-classical, vesicle-mediated secretory pathway triggered by lipopolysaccharide (23). To further investigate this phenomenon, the present study monitored HMGB1 translocation by immunofluorescence. Results demonstrated that HMGB1 was predominantly expressed in the nucleus, while no expression was observed in the cytosol of the normal (sham group) brain. HMGB1 appeared in the cytosol at day 1 after SAH.

HMGB1 is shown to mediate the inflammatory responses through the JAK-STAT signaling pathway. The JAK2/STAT3 signaling pathway is involved in HMGB1 expression in rat peritoneal macrophages after stimulation with $75 \mathrm{ng} / \mathrm{ml} \mathrm{LPS}$ in vitro, while AG490 is able to suppress HMGB1 expression $(11,12)$. Thus, we hypothesized that this may be the potential mechanism involved in the regulation of HMGB1 expression after SAH. The results of the present study demonstrated that JAK2/STAT3 was significantly phosphorylated after SAH, while AG490 significantly inhibited JAK2/STAT3 phosphorylation, further inhibiting the expression of both cytoplasmic and nuclear HMGB1 at day 1 after SAH. Using confocal microscopy, it was demonstrated that AG490 not only decreased the expression of HMGB1, it also reduced cytosol translocation after SAH, which may suppress the pro-inflammatory role of HMGB1 after SAH.

In the present study, inhibition of the JAK2/STAT3 signaling pathway by AG490 was also able to alleviate neuronal apoptosis. Furthermore, brain edema and neurological deficits were both improved by AG490 administration after SAH. Previous 
studies have demonstrated that HMGB1 was involved in the expression of various pro-inflammatory cytokines (24-26). Thus, it was hypothesized that HMGB1 may indirectly induce brain edema by activating the expression of other factors, such as matrix metallopeptidase (MMP)-9 (27), as HMGB1 is able to promote MMP-9 expression. A previous study demonstrated that MMP-9 has an important role in the induction of brain edema (28). Furthermore, the JAK2/STAT3 signaling pathway is activated in cellular and animal models of oxidative stress injury (29). Oxidative stress is able to activate MMPs and fluid channel aquaporins, and shows an important induction of brain edema after brain injury (30). Meanwhile, oxidative stress-induced cell apoptosis and death is directly dependent on JAK2/STAT3 activation (31). Thus, the neuroprotection role of AG490 may also be associated with the inhibition of SAH-induced oxidative stress.

The binding of cytokines and growth factors to glycoprotein 130-associated transmembrane receptors induces the activation of JAK/STAT signaling (32). HMGB1 is able to stimulate the expression of various inflammatory cytokines, including IL-6, IL-1 and TNF- $\alpha$ (33). Thus, it is possible to hypothesize that HMGB1-induced cytokines may induce the activation of the JAK2/STAT3 signaling pathway, which, in turn, results in the expression of HMGB1, activating a vicious circle. However, this hypothesis requires confirmation.

Compared with other types of neurological function rating systems, the Garcia test is more comprehensive and objective, with less bias (17). Thus, the neurological scoring in the present study may effectively indicate the protective effect of AG490 on brain injury after SAH. However, the present study has some limitations. Previous research has indicated that HMGB1 expression after brain damage may induce cerebral vasospasm and delay cerebral ischemia (20). The present study did not investigate the role of HMGB1 in delay cerebral ischemia and it has been demonstrated that HMGB1 is a late pro-inflammatory cytokine (34). The present study demonstrated that HMGB1 increased from $6 \mathrm{~h}$ after SAH onwards, remaining high until day 1 . However, late stages (5 and 7 days) were not investigated in the present study. Therefore, future research should explore the expression of HMGB1 in the late stages of brain damage and its relationship with cerebral ischemia.

In conclusion, the findings herein support the hypothesis that HMGB1 contributes to SAH-induced early brain injury, which is based on the activation of JAK2/STAT3. The JAK2/STAT3 inhibitor, AG490, may be a valuable neuroprotective agent for the treatment of SAH. Targeting HMGB1 and JAK2/STAT3 could be a novel therapeutic strategy applicable for $\mathrm{SAH}$.

\section{References}

1. Suarez JI, Tarr RW and Selman WR: Aneurysmal subarachnoid hemorrhage. N Engl J Med 354: 387-396, 2006.

2. Hansen-Schwartz J, Vajkoczy P, Macdonald RL, Pluta RM and Zhang JH: Cerebral vasospasm: Looking beyond vasoconstriction. Trends Pharmacol Sci 28: 252-256, 2007.

3. Zheng VZ and Wong GKC: Neuroinflammation responses after subarachnoid hemorrhage: A review. J Clin Neurosci 42: 7-11, 2017.

4. Miller BA, Turan N, Chau M and Pradilla G: Inflammation, vasospasm and brain injury after subarachnoid hemorrhage. Biomed Res Int 2014: 384342, 2014.
5. O'Connor KA, Hansen MK, Rachal Pugh C, Deak MM, Biedenkapp JC, Milligan ED, Johnson JD, Wang H, Maier SF, Tracey KJ and Watkins LR: Further characterization of high mobility group box 1 (HMGB1) as a proinflammatory cytokine: Central nervous system effects. Cytokine 24: 254-265, 2003.

6. Faraco G, Fossati S, Bianchi ME, Patrone M, Pedrazzi M, Sparatore B, Moroni F and Chiarugi A: High mobility group box 1 protein is released by neural cells upon different stresses and worsens ischemic neurodegeneration in vitro and in vivo. J Neurochem 103: 590-603, 2007.

7. Cohen MJ, Brohi K, Calfee CS, Rahn P, Chesebro BB, Christiaans SC, Carles M, Howard M and Pittet JF: Early release of high mobility group box nuclear protein 1 after severe trauma in humans: Role of injury severity and tissue hypoperfusion. Crit Care 13: R174, 2009.

8. Ma CX, Yin WN, Cai BW, Wu J, Wang JY, He M, Sun H, Ding JL and You C: Toll-like receptor 4/nuclear factor-kappa B signaling detected in brain after early subarachnoid hemorrhage. Chin Med J (Engl) 122: 1575-1581, 2009.

9. You WC, Wang CX, Pan YX, Zhang X, Zhou XM, Zhang XS Shi JX and Zhou ML: Activation of nuclear factor ${ }_{-} B$ in the brain after experimental subarachnoid hemorrhage and its potential role in delayed brain injury. PLoS One 8: e60290, 2013.

10. Nakahara T, Tsuruta R, Kaneko T, Yamashita S, Fujita M, Kasaoka S, Hashiguchi T, Suzuki M, Maruyama I and Maekawa T: High-mobility group box 1 protein in CSF of patients with subarachnoid hemorrhage. Neurocrit Care 11: 362-368, 2009.

11. Lu B, Antoine DJ, Kwan K, Lundbäck $\mathrm{P}$, Wähämaa $\mathrm{H}$, Schierbeck H, Robinson M, Van Zoelen MA, Yang H, Li J, et al: JAK/STAT1 signaling promotes HMGB1 hyperacetylation and nuclear translocation. Proc Natl Acad Sci USA 111: 3068-3073, 2014.

12. Liu H, Yao YM, Yu Y, Dong N, Yin HN and Sheng ZY: Role of Janus kinase/signal transducer and activator of transcription pathway in regulation of expression and inflammation-promoting activity of high mobility group box protein 1 in rat peritoneal macrophages. Shock 27: 55-60, 2007.

13. Kim OS, Park EJ, Joe EH and Jou I: JAK-STAT signaling mediates gangliosides-induced inflammatory responses in brain microglial cells. J Biol Chem 277: 40594-40601, 2002.

14. De-Fraja C, Conti L, Govoni S, Battaini F and Cattaneo E: STAT signalling in the mature and aging brain. Int J Dev Neurosci 18: 439-446, 2000.

15. Bederson JB, Germano IM and Guarino L: Cortical blood flow and cerebral perfusion pressure in a new noncraniotomy model of subarachnoid hemorrhage in the rat. Stroke 26: 1086-1091, 1995.

16. Damm J, Harden LM, Gerstberger R, Roth J and Rummel C: The putative JAK-STAT inhibitor AG490 exacerbates LPS-fever, reduces sickness behavior, and alters the expression of pro- and anti-inflammatory genes in the rat brain. Neuropharmacology 71: 98-111, 2013.

17. Garcia JH, Wagner S, Liu KF and Hu XJ: Neurological deficit and extent of neuronal necrosis attributable to middle cerebral artery occlusion in rats. Statistical validation. Stroke 26: 627-634, 1995.

18. Sabri M, Ai J, Lakovic K, D'abbondanza J, Ilodigwe D and Macdonald RL: Mechanisms of microthrombi formation after experimental subarachnoid hemorrhage. Neuroscience 224: 26-37, 2012.

19. Dorhout Mees SM, Rinkel GJ, Feigin VL, Algra A, van den Bergh WM, Vermeulen M and van Gijn J: Calcium antagonists for aneurysmal subarachnoid haemorrhage. Cochrane Database Syst Rev: CD000277, 2007.

20. Pradilla G, Chaichana KL, Hoang S, Huang J and Tamargo RJ: Inflammation and cerebral vasospasm after subarachnoid hemorrhage. Neurosurg Clin N Am 21: 365-379, 2010.

21. Murakami K, Koide M, Dumont TM, Russell SR, Tranmer BI and Wellman GC: Subarachnoid hemorrhage induces gliosis and increased expression of the pro-inflammatory cytokine high mobility group box 1 protein. Transl Stroke Res 2: 72-79, 2011.

22. Sun Q, Wu W, Hu YC, Li H, Zhang D, Li S, Li W, Li WD, Ma B, Zhu JH, et al: Early release of high-mobility group box 1 (HMGB1) from neurons in experimental subarachnoid hemorrhage in vivo and in vitro. J Neuroinflammation 11: 106, 2014.

23. Gardella S, Andrei C, Ferrera D, Lotti LV, Torrisi MR, Bianchi ME and Rubartelli A: The nuclear protein HMGB1 is secreted by monocytes via a non-classical, vesicle-mediated secretory pathway. EMBO Rep 3: 995-1001, 2002. 
24. Qiu J, Nishimura M, Wang Y, Sims JR, Qiu S, Savitz SI, Salomone S and Moskowitz MA: Early release of HMGB-1 from neurons after the onset of brain ischemia. J Cereb Blood Flow Metab 28: 927-938, 2008

25. Scaffidi P, Misteli T and Bianchi ME: Release of chromatin protein HMGB1 by necrotic cells triggers inflammation. Nature 418: 191-195, 2002.

26. Kim JB, Sig Choi J, Yu YM, Nam K, Piao CS, Kim SW, Lee MH, Han PL, Park JS and Lee JK: HMGB1, a novel cytokine-like mediator linking acute neuronal death and delayed neuroinflammation in the postischemic brain. J Neurosci 26: 6413-6421, 2006.

27. Qiu J, Xu J, Zheng Y, Wei Y, Zhu X, Lo EH, Moskowitz MA and Sims JR: High-mobility group box 1 promotes metalloproteinase-9 upregulation through Toll-like receptor 4 after cerebral ischemia. Stroke 41: 2077-2082, 2010.

28. Li DD, Song JN, Huang H, Guo XY, An JY, Zhang M, Li Y, Sun P, Pang HG, Zhao YL and Wang JF: The roles of MMP-9/TIMP-1 in cerebral edema following experimental acute cerebral infarction in rats. Neurosci Lett 550: 168-172, 2013.

29. Tawfik A, Jin L, Banes-Berceli AK, Caldwell RB, Ogbi S, Shirley A, Barber D, Catravas JD, Stern DM, Fulton D, et al: Hyperglycemia and reactive oxygen species mediate apoptosis in aortic endothelial cells through Janus kinase 2. Vascul Pharmacol 43: 320-326, 2005.
30. Abdul-Muneer PM, Chandra N and Haorah J: Interactions of oxidative stress and neurovascular inflammation in the pathogenesis of traumatic brain injury. Mol Neurobiol 51: 966-979, 2014.

31. Yu HM, Zhi JL, Cui Y, Tang EH, Sun SN, Feng JQ and Chen PX: Role of the JAK-STAT pathway in protection of hydrogen peroxide preconditioning against apoptosis induced by oxidative stress in PC12 cells. Apoptosis 11: 931-941, 2006.

32. Satriotomo I, Bowen KK and Vemuganti R: JAK2 and STAT3 activation contributes to neuronal damage following transient focal cerebral ischemia. J Neurochem 98: 1353-1368, 2006.

33. Agnello D, Wang H, Yang H, Tracey KJ and Ghezzi P: HMGB-1, a DNA-binding protein with cytokine activity, induces brain TNF and IL-6 production and mediates anorexia and taste aversion. Cytokine 18: 231-236, 2002.

34. Wang $\mathrm{H}$, Bloom O, Zhang M, Vishnubhakat JM, Ombrellino M, Che J, Frazier A, Yang H, Ivanova S, Borovikova L, et al: HMG-1 as a late mediator of endotoxin lethality in mice. Science 285: 248-251, 1999. International (CC BY-NC-ND 4.0) License. 